
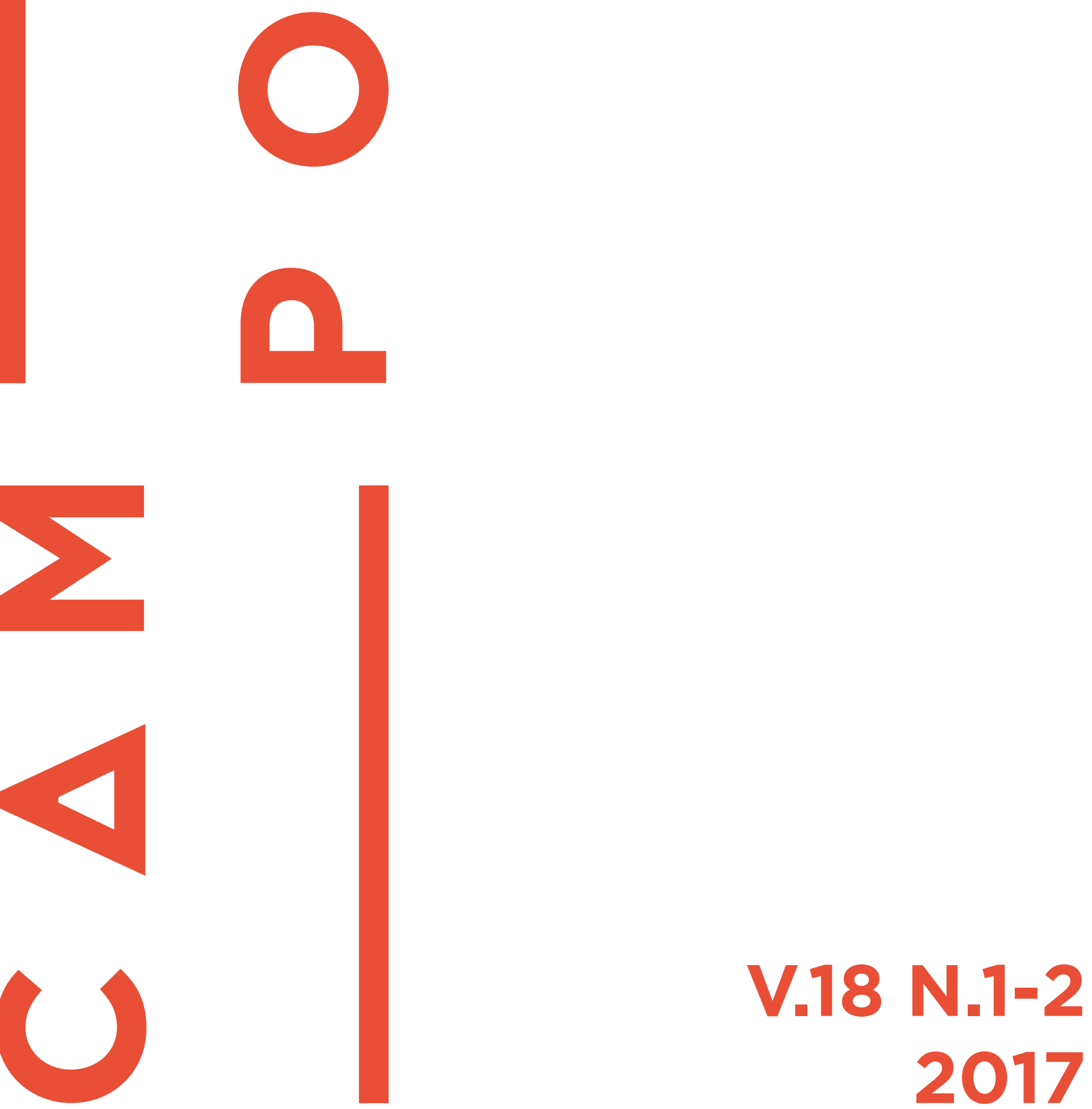

REVISTA DE ANTROPOLOGIA PPGA / UFPR 


\section{Linha Editorial}

CAMPOS - Revista de Antropologia, publicação do Programa de PósGraduação em Antropologia da Universidade Federal do Paraná, tem o propósito de constituir um espaço permanente de interlocução com antropólogos e pesquisadores de áreas afins, no país e no exterior. 0 nome CAMPOS traduz o reconhecimento do valor singular da etnografia para a Antropologia, remetendo também à pluralidade de perspectivas teóricas e temáticas que caracteriza a disciplina e que se revela na produção do próprio PPGA-UFPR. A Revista CAMPOS publica artigos inéditos, ensaios bibliográficos, entrevistas, resenhas e outras contribuições - pequenos textos de natureza acadêmica, informativa etc. - que podem ser enviados em português, inglês, francês ou espanhol (para publicação na língua original).

\section{Editores}

Edilene Coffaci de Lima Patrícia Carvalho Rosa

\section{Comissão Editorial (PPGA-UFPR)}

Ciméa Barbato Bevilaqua

Edilene Coffaci de Lima

Eva Lenita Scheliga

João Frederico Rickli

Laércio Loiola Brochier

Laura Pérez Gil

Liliana Mendonça Porto

Lorenzo Macagno

Marcos Silva da Silveira

Maria Inês Smiljanic

Miguel Alfredo Carid Naveira

Paulo Renato Guérios

Ricardo Cid Fernandes

Sandra Stoll

\section{Conselho Editorial}

América Larraín, Universidad Nacional de Colombia, Colômbia

Aristóteles Barcelos Neto, University of East Anglia, Reino Unido

Birgit Meyer, Utrecht University, Holanda

Christiano Tambascia, UNICAMP, Brasil

Claudia Fonseca, UFRGS, Brasil

Eduardo Viveiros de Castro, MN/ URJ, Brasil

Elena Calvo Gonzalez, UFBA, Brasil

François Laplantine, Université Lumière - Lyon 2, França

José Guilherme Cantor Magnani, USP, Brasil

Lorena Cordoba, CONICET/

Universidad de Buenos

Aires, Argentina e Centro de Investigaciones Históricas y Antropológicas, Bolivia

Manuela Carneiro da Cunha, (University of Chicago, EUA

Márcio Ferreira da Silva, USP, Brasil

Marcio Goldman, MN/UFRJ, Brasil

Martina Ahlert, UFMA, Brasil

Mariza G. S. Peirano, UnB, Brasil

Miriam P. Grossi, UFSC, Brasil

Peter Fry, UFRJ, Brasil

Philippe Erikson, Université de Paris Ouest-Nanterre, França

Rafael José Menezes Bastos, UFSC, Brasil

Roberto DaMatta, UFF/University of Notre Dame, Brasil/EUA

Roque de Barros Laraia, UnB, Brasil

Ruben G. Oliven, UFRGS, Brasil 

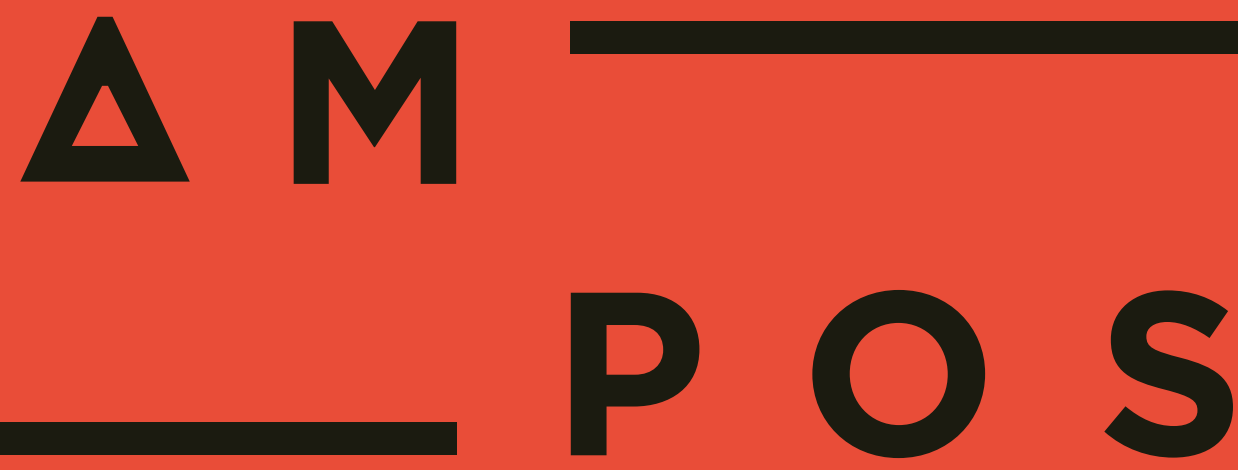

CAMPOS. Revista de Antropologia. PPGA/ UFPR.

V.18 N.1-2 2017

*Publicada em junho 2018

Curitiba, PR, Brasil

ISSN 2317-6830

P. $001-301$

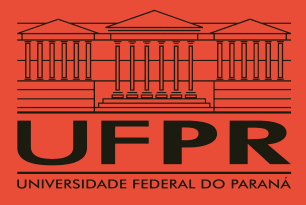


Qualquer parte desta publicação pode ser reproduzida, desde que citada a fonte.

Disponível também em:

revistas.ufpr.br/campos

Publicação indexada em:

Directory of Open Access Journals

- DOAJ (WWw.doaj.org)

Clase - Citas Latinoamericanas en

Ciencias Sociales y Humanidades

(http//132.248.9.1:8991)

Sumários de Revistas Brasileiras

(www.sumarios.org)

Latindex (www.latindex.unam.mx)

\section{Expediente}

\section{Secretaria de Redação}

Edilene Coffaci de Lima

Patrícia Carvalho Rosa

\section{Revisão}

Edilene Coffaci de Lima

Sara Guerreiro Parada

Revisão dos resumos em inglês

Fabiano Atenas Azola
Preparação dos originais

Edilene Coffaci de Lima

\section{Projeto gráfico}

Martim Fernandes

\section{Diagramação}

Rafael Tajima

\section{Redação}

CAMPOS - Revista de Antropologia

Rua General Carneiro, 460 - $6^{\circ}$ andar. CEP 80.060-150 Curitiba Paraná - Brasil

Telefone: (41) 3360-5272

revistacampos.ufpr@gmail.com

CAMPOS: Revista de Antropologia / Universidade Federal do Paraná. Programa de Pós-Graduação em Antropologia ; editores: Ciméa Barbato Bevilaqua [...et al.], n.1 (2001) - Curitiba : UFPR/PPGAS, 2001.

n.18 (1-2), 2017 Semestral

ISSN : $2317-6830$

1.Antropologia Social - Periódicos. I. Universidade Federal do Paraná. Programa de Pós-Graduação em Antropologia Social. II. Bevilaqua, Ciméa Barbato. 


\section{Dossiê - História Intelectual e Etnografia do Fazer Científico}

Introdução ao dossiê História Intelectual e Etnografia do Fazer Científico

Christiano Key Tambascia

o meio de ter ideias imprevistas: Lévi-strauss, fichas e fichários

Luísa Valentini

Um foco de energia: o Collège de sociologie e a noção de sagrado (1937-1939)

Júlia Vilaça Goyatá

Um departamento municipal no ultramar francês: - cosmopolitismo de José Arthur Giannotti

Lidiane Soares Rodrigues

Uma revisão crítica da antropologia de Carmen Junqueira

Lindinalva Laurindo Teodorescu

Trajetórias intelectuais marcadas entre a ciência e a religião: José Loureiro Fernandes e a Faculdade de Filosofia, Ciências e Letras do Paraná

Paulo Guérios

\section{Artigos}

Formas do parentesco: grafismo e enunciação no Vale de Araotz (País Basco)

Ion F. de las Heras

o medo do precedente: as técnicas de decisão no Supremo Tribunal Federal

Andressa Lewandowski 

história de um xuve, seu Calixto Francelino Carolina Perini de Almeida

197
"Politicagem": conclusões a partir das diferenças entre as noções de "administrador" e "politico"

Monique Florencio de Aguiar

Produções Legais da Pirataria: o streaming e a Incorporação das Demandas e Discursos Piratas no Mercado do Entretenimento Digital

Andressa Nunes Soilo

\section{Entrevista}

Da política indigenista às ações afirmativas e à Antropologia do Estado: entrevista com Antonio Carlos de Souza Lima

Edilene Coffaci de Lima, Marcos Silva da Silveira e Lays Gonçalves

\section{Resenhas}

MORAIS, Bruno. 2017. Do corpo ao pó. Crônicas da territorialidade Kaiowá e Guarani nas adjacências da morte. São Paulo: Editora Elefante. 362pp. (Prêmio melhor dissertação de mestrado no concurso Anpocs de obras científicas e teses universitárias em Ciências Sociais). Florbela Almeida Ribeiro

GRACINO JUNIOR, Paulo. 2016. A demanda por deuses: globalização, fluxos religiosos e culturas locais nos dois lados do Atlântico. Rio de Janeiro: EdUERJ. 350 pp. Michelle Gonçalves Rodrigues

SEGATA, Jean; RIFIOTIS, Theophilos (orgs.). 2016. Políticas etnográficas no campo da cibercultura. Brasília, ABA Publicações; Joinville, Editora Letradágua. 208 pp. Madson J Albino Rafael

DULLEY, Iracema. 2015. Os nomes dos outros: etnografia e diferença em Roy Wagner. São Paulo: Humanitas/FAPESP. 251 pp. Esmael Alves de Oliveira 
CAVALCANTI, Maria Laura. 2015. Carnaval, Ritual e Arte. Rio de Janeiro: 7 Letras. 233 pp. Ulisses Corrêa Duarte

279

SOBRAL, L.F. 2015. Bogart duplo de Bogart: pistas da persona cinematográfica de Humphrey Bogart, 1941-1946. São Paulo: Terceiro Nome (Coleção Antropologia Hoje). 152 pp. Diogo Barbosa Maciel

GUERREIRO JUNIOR, Antônio Roberto. 2015. Ancestrais e suas sombras: uma etnografia da chefia Kalapalo e seu ritual mortuário. Campinas, SP: Editora da Unicamp. 520 pp. Thaís Regina Mantovanelli

FERREIRA, Jaqueline e FLEISCHER, Soraya (org.). 2014. Etnografias em serviços de saúde. Rio de Janeiro: Garamond. 360 pp.

Tatiane Vieira Barros

291

Dissertações do Programa de Pós-Graduação em Antropologia da UFPR - 2017

Instruções editoriais para os autores

Diretrizes para autores 


\section{N D E X}

CAMPOS V.18 N.1-2 2017

Dossier - Intellectual History and Ethnography of Scientific Making

11
Intellectual History and Ethnography of Scientific Making Christiano Key Tambascia

The means for having unforseen ideas: LéviStrauss, paper cards and indexes Luísa Valentini

A focus of energy: the Collège of Sociologie and the notion of sacred (1937-1939) Júlia Vilaça Goyatá

A municipal department in French overseas: the cosmopolitanism of José Arthur Gianotti

Lidiane Soares Rodrigues

A critical review of the anthropology of Carmen Junqueira

Lindinalva Laurindo Teodorescu

José Loureiro Fernandes and the Faculty of Philosophy, Sciences and Letters of Paraná: Intellectual trajectories between science and religion

Paulo Guérios

\section{Articles}

Forms of kinship: Graphics and enunciation in the Valley of Araotz (Basque Country) Ion F. de las Heras

The thread of precedent. The techniques of decision in the Federal supreme Court Andressa Lewandowski 
"Politicking": conclusions from the differences between the concepts of "manager" and "politician"

Monique Florencio de Aguiar

Legal Productions of Piracy: Streaming Services and Assimilation of Pirate Demands and Discourses in the Digital Entertainment Market Andressa Nunes Soilo

\section{Interview}

From indigenist policy to affirmative actions and the anthropology of the state: interview with Antonio Carlos de Souza Lima Edilene Coffaci de Lima, Marcos Silva da Silveira e Lays Gonçalves

\section{Reviews}

MORAIS, Bruno. 2017. Do corpo ao pó. Crônicas da territorialidade Kaiowá e Guarani nas adjacências da morte. São Paulo: Editora Elefante. $362 \mathrm{pp}$. (Prêmio melhor dissertação de mestrado no concurso Anpocs de obras científicas e teses universitárias em Ciências Sociais). Florbela Almeida Ribeiro

GRACINO JUNIOR, Paulo. 2016. A demanda por deuses: globalização, fluxos religiosos e culturas locais nos dois lados do Atlântico. Rio de Janeiro: EdUERJ. 350 pp.

Michelle Gonçalves Rodrigues

SEgATA, Jean; RIFIOTIS, Theophilos (orgs.). 2016. Políticas etnográficas no campo da cibercultura. Brasilia, ABA Publicações; Joinville, Editora Letradágua. 208 pp. Madson J Albino Rafael etnografia e diferença em Roy Wagner. São Paulo: Humanitas/FAPESP. 251 pp.

Esmael Alves de Oliveira 

e Arte. Rio de Janeiro: 7 Letras. 233 pp. Ulisses Corrêa Duarte

279

SOBRAL, L.F. 2015. Bogart duplo de Bogart: pistas da persona cinematográfica de Humphrey Bogart, 1941-1946. São Paulo: Terceiro Nome (Coleção Antropologia Hoje). 152 pp. Diogo Barbosa Maciel

283

GUERREIRO JUNIOR, Antônio Roberto. 2015. Ancestrais e suas sombras: uma etnografia da chefia Kalapalo e seu ritual mortuário. Campinas, SP: Editora da Unicamp. 520 pp. Thaís Regina Mantovanelli

287

FERREIRA, Jaqueline e FLEISCHER, Soraya (org.). 2014. Etnografias em serviços de saúde. Rio de Janeiro: Garamond. 360 pp.

Tatiane Vieira Barros

Thesis

299

Instructions to Authors

$* * *$ 